\title{
Scalp hair cortisol for diagnosis of Cushing's syndrome
}

\author{
Vincent L Wester ${ }^{1}$, Martin Reincke ${ }^{4}$, Jan W Koper ${ }^{1}$, Erica L T van den Akker ${ }^{2}$, \\ Laura Manenschijn' ${ }^{1}$, Christina M Berr', Julia Fazel' ${ }^{4}$, Yolanda B de Rijke ${ }^{3}$, \\ Richard A Feelders ${ }^{1}$ and Elisabeth F C van Rossum ${ }^{1}$ \\ ${ }^{1}$ Division of Endocrinology, Department of Internal Medicine, ${ }^{2}$ Department of Pediatrics, ${ }^{3}$ Department \\ of Clinical Chemistry, Erasmus MC, University Medical Center Rotterdam, Rotterdam, the Netherlands, \\ and ${ }^{4}$ Medizinische Klinik und Poliklinik IV, Klinikum der Ludwig-Maximilians-Universität München, \\ Munich, Germany
}

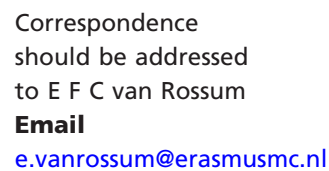

\begin{abstract}
Objective: Current first-line screening tests for Cushing's syndrome (CS) only measure time-point or short-term cortisol. Hair cortisol content (HCC) offers a non-invasive way to measure long-term cortisol exposure over several months of time. We aimed to evaluate HCC as a screening tool for CS.

Design: Case-control study in two academic referral centers for CS.

Methods: Between 2009 and 2016, we collected scalp hair from patients suspected of CS and healthy controls. HCC was measured using ELISA. HCC was available in 43 confirmed CS patients, 35 patients in whom the diagnosis CS was rejected during diagnostic work-up and follow-up (patient controls), and 174 healthy controls. Additionally, we created HCC timelines in two patients with ectopic CS.

Results: CS patients had higher HCC than patient controls and healthy controls (geometric mean 106.9 vs 12.7 and $8.4 \mathrm{pg} / \mathrm{mg}$ respectively, $P<0.001)$. At a cut-off of $31.1 \mathrm{pg} / \mathrm{mg}$, HCC could differentiate between CS patients and healthy controls with a sensitivity of $93 \%$ and a specificity of $90 \%$. With patient controls as a reference, specificity remained the same $(91 \%)$. Within CS patients, HCC correlated significantly with urinary free cortisol $(r=0.691, P<0.001)$. In two ectopic CS patients, HCC timelines indicated that cortisol was increased 3 and 6 months before CS became clinically apparent.

Conclusions: Analysis of cortisol in a single scalp hair sample offers diagnostic accuracy for CS similar to currently used first-line tests, and can be used to investigate cortisol exposure in CS patients months to years back in time, enabling the estimation of disease onset.
\end{abstract}

\section{Introduction}

Glucocorticoids are a class of steroid hormones that are produced under the influence of the hypothalamicpituitary-adrenal (HPA) axis and have mediating effects in metabolism, inflammation, circulation and behavior. Cushing's syndrome (CS) occurs when there is an excess of glucocorticoids, either from an exogenous source or from an excessive endogenous production of cortisol. Endogenous CS is a rare disorder, most commonly caused by an ACTH producing pituitary adenoma (Cushing's disease), less
두 2017 European Society of Endocrinology Printed in Great Britain frequent are primary adrenal causes and ectopic CS (1). Ectopic CS results from secretion of adrenocorticotropic hormone (ACTH) from a non-pituitary source, or very rarely, from ectopic corticotropin-releasing hormone (CRH) secretion. Ectopic CS is often associated with higher ACTH levels than in Cushing's disease, leading to more fulminant CS (2). While a number of signs and symptoms are deemed highly suggestive for CS (e.g. facial plethora, proximal muscle weakness, purple striae and

Published by Bioscientifica Ltd. 
easy bruising), many are non-specific and highly prevalent in the general population, such as metabolic syndrome features, osteoporosis and depression (3). This overlap with common chronic conditions is likely to cause a delay in the diagnosis of CS in many cases.

Diagnosis of CS is further complicated by the fact that no single biochemical test for cortisol exposure offers perfect diagnostic accuracy. First-line tests for CS that are recommended by the Endocrine Society guideline are urinary free cortisol (UFC) in 24-h urine collections, latenight salivary cortisol (LNSC) and the $1 \mathrm{mg}$ overnight dexamethasone suppression test (DST) (3). Cortisol secretion can be variable in CS, illustrated by the fact that there is a high variability of UFC in patients with active CS (4) and that many patients have at least one normal UFC (5). In general, multiple tests are needed to establish a diagnosis, and thus, both UFC and LNSC measurements are usually performed on at least two different occasions (3).

Over the past few years there is an increasing use of cortisol in scalp hair as a measure of long-term cortisol exposure (6). Hair cortisol content (HCC) has been used to investigate long-term cortisol levels in association with cardiovascular disease $(7,8,9)$, obesity $(10,11,12)$ and metabolic syndrome (13). We and others have previously reported increased HCC in a small number of CS patients, including cases of cyclic CS $(14,15)$. HCC has practical advantages over currently used diagnostic tests, since sample collection can easily be performed in an outpatient setting and is not dependent on patient adherence to sampling instructions. Furthermore, HCC measurement offers retrospective information about cortisol levels over months of time in a single measurement, thereby potentially circumventing the limitations posed by the variability in cortisol secretion in endogenous CS (6).

We aimed to establish the optimal cut-off value of HCC for the diagnosis of endogenous CS. In order to do this, we measured HCC in patients with confirmed CS, in healthy controls and in patients who were initially suspected to have CS but in whom CS was excluded during work-up. Furthermore, we aimed to explore the potential of HCC to retrospectively assess the onset of hypercortisolism in severe (ectopic) CS.

\section{Subjects and methods}

\section{Patients and controls: HCC for diagnosis of Cushing's syndrome}

Between 2009 and 2016, patients who visited the endocrinology outpatient clinic at a single academic medical center (Erasmus MC, Rotterdam, the Netherlands) and were suspected of CS were requested to take part in the present study. Within this period, we included all patients who were evaluated for Cushing's syndrome and consented to take part in the present study, and in whom Cushing's syndrome could either be confirmed, or excluded based on the diagnostic tests and clinical evaluation of the treating endocrinologist. Part of these patients were included in a previously published case series (15). We excluded patients who used topical hydrocortisone, as well as users of systemic corticosteroids. Patients were classified as having endogenous CS if hypercortisolism could be biochemically confirmed in the three months before study inclusion (using UFC, DST and/or LNSC), and the cause of hypercortisolism could be demonstrated. For Cushing's disease, the combination of hypercortisolism with an inferior petrosal sinus sampling showing a central to peripheral ACTH gradient, or a pituitary adenoma $>6 \mathrm{~mm}$ on MRI was considered diagnostic. For adrenal Cushing's syndrome, histopathology of a cortisolproducing adenoma was considered diagnostic. For ectopic Cushing's syndrome, histopathology of an ACTH or CRH producing tumor was considered diagnostic. Healthy individuals from our previously published validation study, which were included from August 2009 through April 2010, served as controls (16). From all participants, written informed consent was obtained. This study was approved by the institutional review board of Erasmus MC. Using questionnaires, information about hair characteristics, which have been shown to influence hair cortisol, frequency of hair washing, the use of hair products and hair treatments like coloring and bleaching were obtained from all participants. Furthermore, this questionnaire included a question about the recent use of corticosteroids, including topical corticosteroids (6).

\section{Patients: HCC timelines in ectopic Cushing's syndrome}

Two patients with ectopic CS and long hair were recruited at the Klinikum der Ludwig-Maximilians-Universität München (Munich, Germany) with the objective to create retrospective timelines of cortisol exposure. From both the participants, written informed consent was obtained. This study was approved by the institutional review board of the Klinikum der Ludwig-Maximilians-Universität München.

\section{Measurement of hair cortisol concentrations}

In all participants we cut a scalp hair sample of approximately 150 hairs at the posterior vertex, as close 
to the scalp as possible. Hair processing and analysis was performed as described previously (16). Depending on the length of the hair, from the most proximal $1-3 \mathrm{~cm}$ at least $10 \mathrm{mg}$ of hair was weighed. For the creation of timelines in patients with ectopic CS, the entire length of hair samples was divided into segments of $1 \mathrm{~cm}$ length, corresponding to cortisol exposure during periods of 1 month (17). Depending on the quantity of hair, more distal hair was divided in $2 \mathrm{~cm}$ segments, corresponding to 2-month periods.

After weighing, the hair was finely cut using scissors. We extracted cortisol from the hair in $1 \mathrm{~mL}$ of methanol during $16 \mathrm{~h}$ at $52^{\circ} \mathrm{C}$. After extraction, the methanol was transferred into clean glass tubes, evaporated under nitrogen stream and the residue was reconstituted in $250 \mu \mathrm{L}$ of phosphate buffered saline ( $\mathrm{pH}$ 8.0). We then vortexed the samples and analyzed them using a commercially available ELISA kit for cortisol in saliva (SLV-2930, DRG Instrument GmbH, Marburg, Germany). We previously determined the intra- and inter-assay variations. For the intra-assay variation, coefficients of variance $(\mathrm{CV})$ were $3.1 \%$ at $4.4 \mathrm{ng} / \mathrm{mL}, 2.3 \%$ at $21.3 \mathrm{ng} /$ $\mathrm{mL}$ and $2.6 \%$ at $35.0 \mathrm{ng} / \mathrm{mL}$. The inter-assay CVs were 7.0 , 2.3 and $8.2 \%$ respectively (18).

\section{Measurement of cortisol concentrations in urine, serum and saliva}

Twenty-four-hour UFC was measured on two consecutive days as a part of the routine diagnostic procedure for CS. UFC was measured using either one of two in-house methods: chemiluminescence immunoassay using unextracted urine (Immulite XPi, Siemens AG) or liquid chromatography/tandem mass spectrometry (LC/MS-MS, Waters Xevo-TQ-S, Milford, MA, USA). The upper limits of normal of these assays are 850 (validated for cortisol production rate) and $133 \mathrm{nmol} / 24 \mathrm{~h}$ respectively.

Serum cortisol was measured using chemiluminescence immunoassay (Immulite XPi, Siemens AG). A morning cortisol after $1 \mathrm{mg}$ dexamethasone overnight of more than $50 \mathrm{nmol} / \mathrm{L}$ suggested hypercortisolism. Salivary cortisol was measured using ELISA (SLV-2930, DRG Instrument GmbH, Marburg, Germany, or DES6611, Demeditec Diagnostics GmbH, Kiel-Wellsee, Germany). For LNSC, a cut-off value of $9.3 \mathrm{nmol} / \mathrm{L}$ was used, as reported previously (19).

In the two patients with ectopic CS from the Klinikum der Ludwig-Maximilians-Universität München, serum cortisol was measured using solid phase antigen linked technique (Liaison, DiaSorin Deutschland GmbH,
Dietzenbach, Germany), and salivary cortisol was measured using a luminescence immunoassay (Cortisol Luminescence Immunoassay, IBL International $\mathrm{GmbH}$, Hamburg, Germany). UFC was measured using two different chemiluminescence immunoassays for patient A (DVIA Centaur, Siemens AG) and patient B (Liaison, DiaSorin Deutschland GmbH, Dietzenbach, Germany).

\section{Statistical analysis}

IBM SPSS Statistics version 21 (IBM) and GraphPad Prism version 5.01 (GraphPad Software) were used for statistical analysis. Baseline characteristics were analyzed using chi square, Mann-Whitney $U$ and Kruskall-Wallis tests. Cortisol values were logarithmically transformed to obtain a normal distribution. HCC is expressed as a geometric mean and 95\% confidence interval, in $\mathrm{pg} / \mathrm{mg}$ hair. We compared HCC between CS patients, healthy controls and non-CS patients with analysis of (co)variance. To determine cut-off values, sensitivity and specificity, we created receiver operating characteristic (ROC) curves. Correlations between HCC and first-line screening tests in CS patients were analyzed using Pearson's correlation.

\section{Results}

\section{Diagnosis accuracy of HCC in CS}

We included 43 patients with confirmed endogenous CS, including patients from a previously published case series (15). In addition, we identified 35 patients who had suspected CS, but in whom CS diagnosis was excluded during diagnostic work-up (patient controls: median follow-up 3.5 months, range: 0.5-91.2). All 35 patient controls had UFCs below the upper limit of normal. LNSC values were measured in 33 out of 35 patient controls, and were all below the cut-off value of $9.3 \mathrm{nmol} / \mathrm{L}$. Patients with CS were on average older than the 174 healthy controls (median age 50 (range: 15-76) vs 32 years (range: $18-63$ ) $P<0.001$ ) and had a higher BMI (29.4 (range: $18.3-81.6$ ) vs $23.5 \mathrm{~kg} / \mathrm{m}^{2}$ (range: 16.9 43.3), $P<0.001$, Table 1). CS patients had lower BMI than patient controls (29.4 (range: 18.3-81.6) vs 35.2 (range: $\left.21.4-46.6) \mathrm{kg} / \mathrm{m}^{2}, P=0.011\right)$ and were significantly older (50 (range: $15-76)$ vs 38 (15-79 years), $P=0.009$ ). The group of healthy controls consisted mostly of men, followed by CS patients and patient controls (43 vs 30 vs $17 \%, P=0.011)$. The three groups were similar in terms of hair characteristics (Table 1). HCC levels were highest in CS patients (geometric mean: $106.9 \mathrm{pg} / \mathrm{mg}$, 
Table 1 Baseline characteristics and hair cortisol content. Differences in baseline characteristics were analyzed using chi square and Kruskal-Wallis tests. Differences in hair cortisol content were analyzed using analysis of (co)variance. Hair cortisol data for healthy controls, and part of that of CS patients have been published previously $(15,16)$.

\begin{tabular}{l}
\hline \\
\hline Patient characteristics \\
Male $(n, \%)$ \\
Age (years) (median, range) \\
BMI (kg/m²) (median, range) \\
Hair characteristics \\
Hair washing $\geq 3$ per week (yes/no, \%) \\
Use of hair products (yes/no, \%) \\
Hair coloring* (yes/no, \%) \\
Hair bleaching* (yes/no, \%) \\
Hair cortisol content \\
Non-adjusted (pg/mg hair) (geometric mean, 95\% Cl) \\
Adjusted model $1^{\dagger}(\mathrm{pg} / \mathrm{mg}$ hair) (geometric mean, 95\% Cl) \\
Adjusted model $2^{\ddagger}(\mathrm{pg} / \mathrm{mg}$ hair) (geometric mean, $95 \% \mathrm{Cl}$ ) \\
First-line screening tests \\
Urinary free cortisol (ULN) (geometric mean, 95\% Cl) \\
Late-night salicary cortisol (nmol/L) (geometric mean, \\
$95 \%$ Cl) \\
DST cortisol (nmol/L) (geometric mean, 95\% Cl)
\end{tabular}

\begin{tabular}{c}
$\begin{array}{c}\text { Healthy controls, } \\
n=174\end{array}$ \\
\hline $74(43 \%)$ \\
$32(18-63)$ \\
$23.5(16.9-43.3)$ \\
\\
$127 / 45(74 \%)$ \\
$84 / 89(49 \%)$ \\
$36 / 138(21 \%)$ \\
$13 / 161(7 \%)$ \\
$8.4(7.0-10.0)$ \\
$8.3(6.7-10.3)$ \\
$8.6(6.9-10.6)$
\end{tabular}

\begin{tabular}{c}
$\begin{array}{c}\text { Patient controls, } \\
n=35\end{array}$ \\
\hline $6(17 \%)$ \\
$38(15-79)$ \\
$35.2(21.4-46.6)$ \\
$25 / 9(74 \%)$ \\
$17 / 17(50 \%)$ \\
$13 / 21(38 \%)$ \\
$4 / 30(12 \%)$ \\
$12.7(8.6-18.6)$ \\
$13.8(8.6-21.8)$ \\
$15.2(9.5-24.1)$ \\
$0.42(0.31-0.57)$ \\
$2.2(1.4-3.5)$ \\
$40(30-54)$ \\
\hline
\end{tabular}

\begin{tabular}{|c|c|}
\hline $\begin{array}{c}\text { CS patients, } \\
n=43\end{array}$ & $\boldsymbol{P}_{\text {diff }}$ \\
\hline $13(30 \%)$ & 0.011 \\
\hline $50(15-76)$ & $<0.001$ \\
\hline $29.4(18.3-81.6)$ & $<0.001$ \\
\hline $24 / 16(60 \%)$ & 0.209 \\
\hline $12 / 28(30 \%)$ & 0.091 \\
\hline $10 / 33(23 \%)$ & 0.088 \\
\hline $1 / 42(2 \%)$ & 0.270 \\
\hline 106.9 (77.1-147.9) & $<0.001$ \\
\hline $95.8(66.6-137.6)$ & $<0.001$ \\
\hline $93.0(63.7-135.6)$ & $<0.001$ \\
\hline $3.81(2.87-5.05)$ & $<0.001$ \\
\hline $27.7(18.1-42.4)$ & $<0.001$ \\
\hline 476 (369-615) & $<0.001$ \\
\hline
\end{tabular}

*Hair coloring and bleaching in the 3 months prior to hair collection. ${ }^{\dagger}$ Model 1 was adjusted for age, sex and BMI. ${ }^{\ddagger}$ Model 2 was adjusted for age, sex, $\mathrm{BMI}$ and hair characteristics.

$\mathrm{BMI}$, body mass index; $\mathrm{Cl}$, confidence interval; CS, Cushing's syndrome; DST, dexamethasone suppression test; ULN, upper limit of normal.

95\% CI: 77.1-147.9, $F(2,249)=88.9, \quad P<0.001)$, and significantly higher than in healthy controls $(8.4 \mathrm{pg} /$ mg, 95\% CI: 7.0-10.0, $P<0.001)$ and patients controls (12.7 pg/mg, 95\% CI: 8.6-18.6, $P<0.001)$. Adjustment for age, sex and BMI and hair characteristics did not change these results (Table 1 ). In healthy controls, HCC was not significantly influenced by sex $(t(172)=1.05$, $P=0.293$ ), age (Pearson's $r=0.65, P=0.395$ ) or BMI (Pearson's $r=0.111, P=0.180$ ).

The ROC curve revealed an optimal cut-off for the diagnosis of CS of $31.1 \mathrm{pg} / \mathrm{mg}$ hair, when healthy controls were used as a reference population. For this cut-off, sensitivity and specificity were 93 and 90\% respectively (AUC $=0.958$, Fig. 1A). When we used patient controls as a reference, the optimal cut-off for the diagnosis was the same as with healthy controls. In this analysis, sensitivity and specificity remained similar at 93\% and 91\% respectively (AUC $=0.951$, Fig. 1B).

In CS patients, HCC levels significantly correlated with UFC (available in $n=41$, Pearson's $r=0.691, P<0.001$, Fig. 2). HCC also correlated with serum cortisol after $1 \mathrm{mg}$ DST $(n=25, r=0.724, P<0.001)$ and with LNSC $(n=33$, $r=0.761, P<0.001)$.

Most CS patients had Cushing's disease $(n=26$, $60 \%)$, followed by adrenal CS $(n=10,23 \%)$ and ectopic ACTH secretion $(n=7,16 \%)$. HCC was significantly higher in patients with ectopic ACTH secretion (geometric mean: 412.5 (95\% CI: 176.7-961.1) pg/mg) compared to patients with Cushing's disease and adrenal CS (82.6 (95\% CI: 53.0-128.6) and 80.5 (95\% CI: 39.2-164.3) pg/mg respectively. $F(2,40)=6.18$, $P=0.005$, both pairwise comparisons vs ectopic ACTH secretion $P<0.01$, Fig. 3). Within Cushing's disease patients, the 6 patients with a macroadenoma had a significantly higher HCC than the 20 patients with
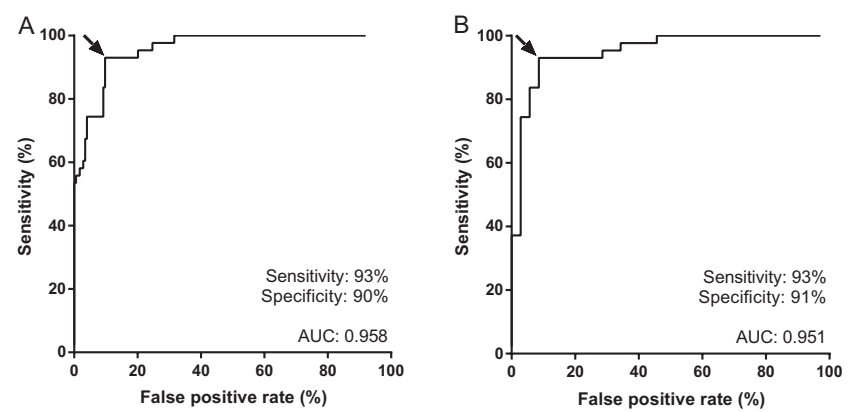

Figure 1

ROC curves of HCC for the diagnosis of CS, with healthy controls $(A)$ or patient controls $(B)$ as a reference population. The arrows indicate a cut-off value of $31.1 \mathrm{pg} / \mathrm{mg}$. AUC, area under the curve. ${ }^{*} P<0.01$. Hair cortisol data for healthy controls, and part of the CS patients have been published previously $(15,16)$. 

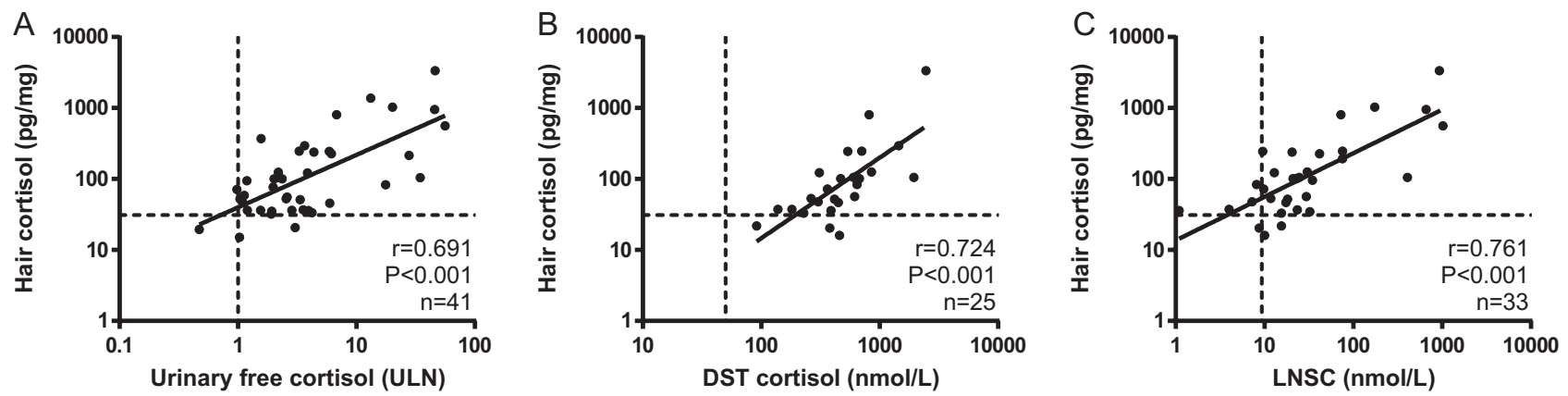

\section{Figure 2}

Correlation between hair cortisol and first-line diagnostic tests in Cushing's syndrome patients. Panel A, urinary free cortisol (UFC). Panel B, dexamethasone suppression test (DST). Panel C, late-night salivary cortisol (LNSC). Hair cortisol content is expressed on a logarithmic scale, in pg/mg hair. UFC is expressed in times upper limit of normal (ULN) on a logarithmic scale. The horizontal dotted line represents the cut-off value for hair cortisol of $31.1 \mathrm{pg} / \mathrm{mg}$. The vertical dotted line represents the upper limit of normal for first-line diagnostic test. R, Pearson's correlation. Hair cortisol data for healthy controls, and part of the CS patients have been published previously $(15,16)$.

a microadenoma (227.2 (98.2-524.2) vs 60.9 (44.8$82.6) \mathrm{pg} / \mathrm{mg}, t(24)=3.63, P=0.001)$.

\section{Hair cortisol timelines in patients with ectopic CS}

Patient A was a 54-year old woman, presented at the Klinikum der Ludwig-Maximilians-Universität in Munich in October 2014 with severe hypokalemic hypertension (minimum serum potassium $=1.8 \mathrm{mmol} / \mathrm{L}$ ) and edema, for evaluation of mineralocorticoid excess. However, plasma renin concentration ( $2 \mathrm{U} / \mathrm{L}$, normal: 4.4-46) and plasma aldosterone (<83 pmol/L, normal: 139-979 pmol/L) were suppressed. Before October 2014, she was asymptomatic. Classical signs and symptoms of CS were absent. ACTHdependent hypercortisolism was found, with a UFC of $44358 \mathrm{nmol} / 24 \mathrm{~h}$ (normal: $<414 \mathrm{nmol} / 24 \mathrm{~h}$ ), and a baseline serum cortisol of $3862 \mathrm{nmol} / \mathrm{L}$ (normal: 138-690 nmol/L). The patient underwent $\mathrm{CRH}$ stimulation testing, lowand high-dose dexamethasone testing, and sinus petrosal inferior sampling suggesting an ectopic ACTH source, but intensive imaging studies including FDG PET, DOTATATE PET and DOPA PET scanning were negative. The patient underwent emergency bilateral adrenalectomy after controlling hypercortisolism by CYP11B2 blockade using intravenous etomidate. The source of ACTH has not been detected thus far (last follow-up June 2016).

In the middle of November 2014, approximately 4 weeks after the onset, a hair sample was obtained, with a length of $26 \mathrm{~cm}$, allowing us to retrospectively assess cortisol levels for over 2 years back in time (26 months; Fig. 4A). The most proximal $12 \mathrm{~cm}$ of hair, which correspond with the period from November 2013 to November 2014, were divided into $1 \mathrm{~cm}$ segments. The most distal $14 \mathrm{~cm}$ of hair were divided into $2 \mathrm{~cm}$ long segments, spanning the period between September 2012 and November 2013. Between April 2013 and April 2014, HCC values in Patient A were around the previously established cut-off value $(<31.1 \mathrm{pg} / \mathrm{mg})$. From April 2014 onward, 6 months before clinical presentation, a gradual rise in HCC can be observed, with a maximum of $78.2 \mathrm{pg} /$ $\mathrm{mg}$. In the hair segments corresponding to the fall of 2012, increased HCC can be observed as well (Fig. 4A). Patient A did not recall any symptoms of CS during this period.

Patient B was a 59-year-old woman, presented in September 2015 at the Klinikum der LudwigMaximilians-Universität in Munich with symptoms of rapid weight gain ( $7 \mathrm{~kg}$ in 2 weeks), edema, abdominal obesity, plethora, hirsutism, hair loss, proximal muscle weakness, irritability and polydipsia. All symptoms had a recent onset of less than one month earlier. Clinical examination showed plethora, dorsal fat pad, proximal muscle weakness, edema of the lower extremities, moon face, abdominal obesity, hirsutism, alopecia and dry skin. ACTH-dependent hypercortisolism was found, with a LNSC of $308 \mathrm{nmol} / \mathrm{L}$ (normal: $<4.1 \mathrm{nmol} / \mathrm{L}$ ), and a UFC of $11361 \mathrm{nmol} / 24 \mathrm{~h}$ (normal: $<229 \mathrm{nmol} / 24 \mathrm{~h}$ ). A CRH test, high-dose dexamethasone suppression test and inferior sinus pretrosal sampling were all in accordance with an ectopic Cushing's syndrome. On PET-CT, a suspected pancreatic lesion was seen, but this was not evident from an abdominal MRI and endoscopic sonography 


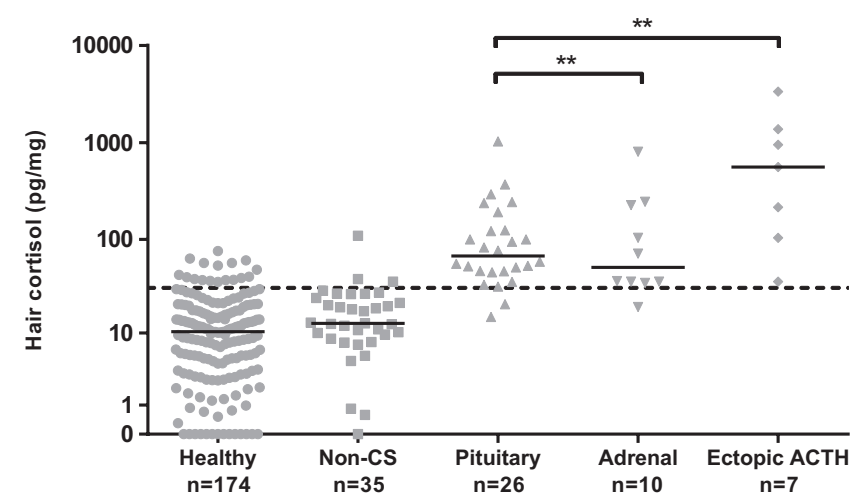

Figure 3

Hair cortisol concentrations in healthy controls, patients without Cushing's syndrome (Non-CS patients), and in patients with Cushing's syndrome. Hair cortisol values are presented on a semi-logarithmic scale. Values in patients with Cushing's syndrome are stratified by etiology. Solid horizontal lines represent group medians, the dotted horizontal line represents the cut-off value of $31.1 \mathrm{pg} / \mathrm{mg}$. Hair cortisol data for healthy controls, and part of the CS patients have been published previously $(15,16)$.

that followed. Based on a diagnosis of occult ectopic Cushing's syndrome, adrenostatic therapy was started in December 2015, with ketoconazole, metyrapone and hydrocortisone substitution. After this, the patient became normocortisolemic. In June 2016, UFC was $151 \mathrm{nmol} / 24 \mathrm{~h}$ (normal: <229/24h).

In November 2015, slightly over 2 months after the onset of symptoms, a hair sample of $11 \mathrm{~cm}$ length was obtained, allowing us to create a retrospective timeline spanning December 2014 to November 2015 (Fig. 4B). The most proximal $7 \mathrm{~cm}$ of hair was divided into $1 \mathrm{~cm}$ long segments, while the most distal $4 \mathrm{~cm}$ was divided into two $2 \mathrm{~cm}$ long segments. In hair segments corresponding to December 2014 to June 2015, cortisol levels appear below or just above the cut-off level $(<31.1 \mathrm{pg} / \mathrm{mg})$. From June 2015, 3 months before the onset of symptoms, cortisol levels raised sharply, up to $311.2 \mathrm{pg} / \mathrm{mg}$.

\section{Discussion}

In the present study, we used HCC to distinguish between individuals with CS, and those without. We found that a cut-off value of $31.1 \mathrm{pg} / \mathrm{mg}$ discriminates well between CS and healthy controls. Additionally, we showed that this test performs equally well when CS patients are compared
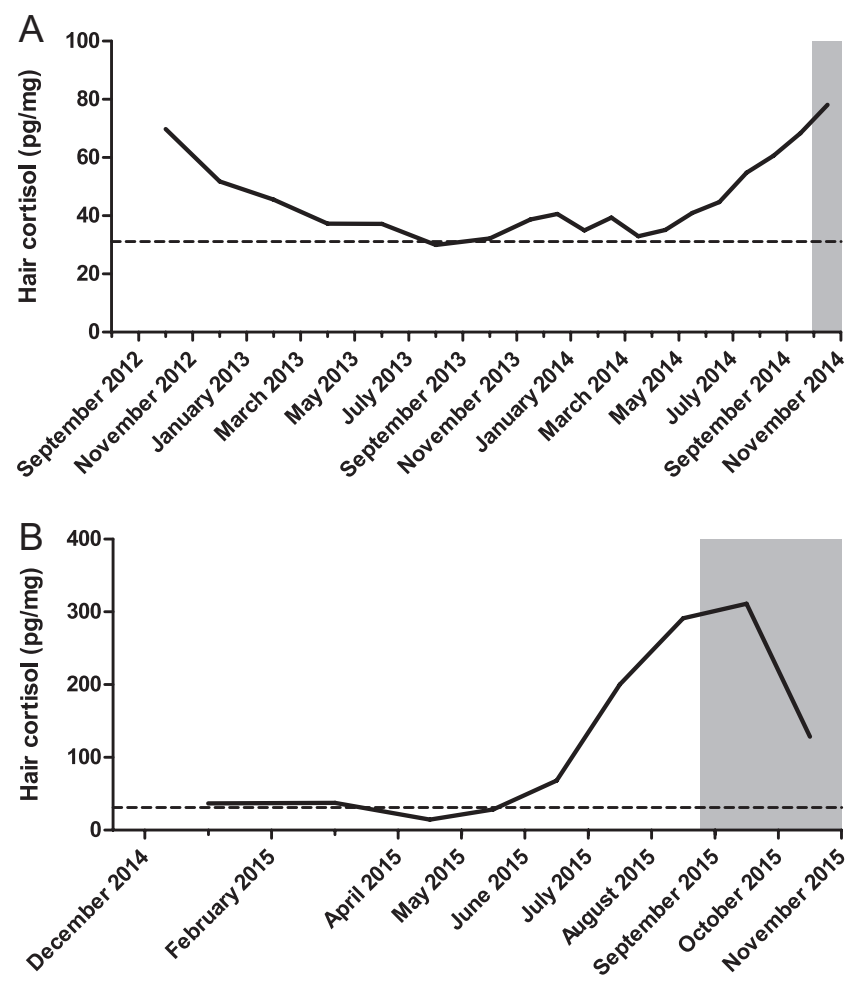

Figure 4

Hair cortisol timelines in patients with a recent onset of ectopic Cushing's syndrome. The horizontal dotted line represents the cut-off value of $31.1 \mathrm{pg} / \mathrm{mg}$. The gray area corresponds to period in which the patient had signs and/or symptoms of Cushing's syndrome.

to patients who were suspected to have CS, but in whom the diagnosis could be excluded (patient controls). The sensitivity of $93 \%$ and specificity of $90 \%$ (healthy controls) and 91\% (patient controls) we found are well in line with current first-line tests for CS as reported in recent meta-analyses: UFC (mean sensitivity and specificity of 84 and 92\% respectively) (20), LNSC (95 and 92\%) (21) and $1 \mathrm{mg}$ DST (99 and 88\%) (20). However, it must be noted that the performance of first-line test differs significantly between studies.

In two patients with recent onset of ectopic CS, we created retrospective timelines of cortisol exposure. In both cases an increase can be seen months before the effects of hypercortisolism became clinically evident. In the case of patient $A$, the hair sample surprisingly seems to indicate hypercortisolism as much as 2 years before hair collection. This may indicate that she has suffered from a previous period of hypercortisolism and thus a cyclical form of CS, although the patient did not recall symptoms of CS around that period. We previously showed that hair 
cortisol can well reveal cyclical forms of Cushing's with more than one period of symptomatic hypercortisolism in the past (15). Care must be taken not to draw exaggerated conclusions based on hair cortisol timelines, especially in the lack of supporting evidence. However, these cases do illustrate the potential of HCC measurements, offering the unique possibility to retrospectively assess cortisol levels months up to years back in time (6). The use of HCC as a historical record of Cushing's syndrome was first shown by Thompson et al. (14), and we have published additional examples in which HCC timelines corresponded well with the clinical course in Cushing's (15). Now, for the first time we showed that the onset of hypercortisolism due to ectopic ACTH production could be estimated. From this, we may learn in the future how the duration of the disease (before diagnosis) may affect the somatic and neuropsychological outcomes of the disease both in short-term and, importantly, in long-term after curation.

Besides the addition of retrospective long-term cortisol measurement, scalp hair cortisol offers significant advantages over current first-line tests for hypercortisolism. First, hair sampling can easily be performed in an inpatient or outpatient setting. Unlike all of the other first-line tests, it does not depend on the adherence of patients to instructions concerning sample collection or medication use. Obviously, complete baldness makes scalp hair sample collection impossible, but in our experience, even in individuals with little scalp hair a useful sample can be obtained successfully. Second, our results are based on collecting just one hair sample. Currently, often multiple urine or saliva collections are required to establish the diagnosis of CS $(3,5)$. Recently, it has been shown that HCC in the proximal $1 \mathrm{~cm}$ of scalp hair (representative of the month before sample collection) correlates well with integrated daily salivary cortisol, based on three daily saliva samples, which were collected for a 30-day period (22). This is strong evidence that cortisol in hair truly reflects the average long-term exposure to cortisol, which dampens the effects of the incidental fluctuations (such as stress at the moment of sample collection) that seriously affect the concentrations that are measured in the other tests.

Although the measurement of scalp hair cortisol is more laborious than cortisol measurements in other matrices, due to hair sample preparation and extraction, it requires no sophisticated techniques. Hair cortisol measurements have been performed for years by several expertise centers $(14,16,23)$. A previously published round robin comparison between hair cortisol methods from different labs found that different methods correlated well, with $r^{2}$ values between 0.88 and 0.97 (24). Further standardization of analytical procedures, as well as automation of hair sample work-up may help routine laboratories set up hair cortisol assays. Like any analytical method hair cortisol relies on proper training of laboratory personnel, in order to limit operator variability. Furthermore, when using any immunoassay based method for an extended period of time, there is a risk of assay performance drift. In our lab, we have consistently used the same equipment and ELISA kits. However, we do rely on quality controls procedures as performed by the manufacturing company.

Several limitations have to be considered when interpreting our data. We included patients in our study, who either had unequivocally confirmed CS, and patients in whom it could unequivocally be excluded. Since the diagnosis of CS was based on current first-line tests, our data do not allow a comparison between the diagnostic accuracy of HCC and these other tests. Additionally, we did not investigate the added value of hair cortisol in cases in which the diagnosis of CS remains unclear. Future prospective studies may show the additional value of HCC in these cases.

Experience with current first-line tests for CS illustrates the importance of investigating possible confounders of screening tests for CS. UFC may be falsely elevated due to high urine volumes and stress, and falsely low with impaired renal function. The DST is subjected to the absorption and metabolism of dexamethasone, which can be influenced by medication use. Furthermore, as total levels of cortisol are measured in serum, the DST is often false positive when medication is used that increases the levels of corticosteroid binding globulin, such as oral contraceptives. LNSC can be falsely elevated due to stress, an altered day rhythm (e.g. in shift workers) and contamination by blood or corticosteroid containing products (3). In our study we did not include any patients who used topical hydrocortisone. However, this is likely to be a confounder, which should be taken into account when interpreting hair cortisol concentrations, similar to LNSC (25). Since HCC estimates cortisol over a much larger timeframe than other tests, the influence of acute stress can be assumed to be diminished.

Just like with measurements in other matrices, many conditions that slightly increase cortisol in scalp hair have now been described. These include depression and obesity $(10,11,12,23)$, which are also common features seen in CS. Therefore, we believe it remains of vital importance 
that the use of HCC for the diagnosis of CS is limited to populations with a high a priori probability. As we have shown in our comparison with patient controls, HCC performed well at diagnosing CS in a population where the suspicion for this diagnosis was high. However, not all factors contributing to HCC may be known yet. Furthermore, in the current study we did not have data on ethnicity, which has been reported to slightly influence hair cortisol (26).

In conclusion, our results indicate that measuring cortisol in a single sample of scalp hair offers a high sensitivity and specificity for the diagnosis of Cushing's syndrome (CS). Together with a straightforward sample collection procedure, this method may prove to be a convenient non-invasive screening test for CS. Additionally, our results indicate that hair cortisol measurements provide clinicians a tool to retrospectively assess cortisol secretion in patients with CS, months to years back in time. This also offers the opportunity to estimate the onset of hypercortisolism and thus the duration of the disease before diagnosis. Future studies using retrospective timelines of hair cortisol may lead to more insight into the question how the total duration of hypercortisolism may affect both somatic and neuropsychological outcomes of the disease in the shortand long-term.

\section{Declaration of interest}

The authors declare that there is no conflict of interest that could be perceived as prejudicing the impartiality of this study.

Funding

Prof. Elisabeth F C van Rossum is supported by an Erasmus MC fellowship grant and the Netherlands Organization for Scientific Research (NWO) grant number 916.96.069. Prof. Martin Reincke is supported by the Else Kröner Fresenius Stiftung (German Cushing's Registry; 2013_A182 and 2015_A228).

\section{Author contribution statement}

V L W, R A F and E F C v R designed the study. V L W, M R, E L T V d A, C $M B, J F, R A F$ and E F C V R cared for the patients and obtained clinical information. $V$ L W and L M performed the hair cortisol measurements. J W K and $Y B$ d R supervised the cortisol measurements. V L W analyzed the data, and wrote the first draft of the manuscript. All authors contributed to the interpretation of the data and writing of the manuscript, and approved the final version of the manuscript.

\section{Acknowledgements}

The authors would like to express their gratitude to Fadime Dogan, Sabine M Staufenbiel and Gerard Noppe for their help in sample workup and analysis, and to Nancy Knossenburg and Stephanie Zopp for their assistance in collecting the hair samples.

\section{References}

1 Lindholm J, Juul S, Jorgensen JO, Astrup J, Bjerre P, Feldt-Rasmussen U, Hagen C, Jorgensen J, Kosteljanetz M, Kristensen L et al. Incidence and late prognosis of Cushing's syndrome: a population-based study. Journal of Clinical Endocrinology and Metabolism 200186 117-123. (doi:10.1210/jc.86.1.117)

2 Lacroix A, Feelders RA, Stratakis CA \& Nieman LK. Cushing's syndrome. Lancet 2015386 913-927. (doi:10.1016/S01406736(14)61375-1)

3 Nieman LK, Biller BM, Findling JW, Newell-Price J, Savage MO, Stewart PM \& Montori VM. The diagnosis of Cushing's syndrome: an Endocrine Society Clinical Practice Guideline. Journal of Clinical Endocrinology and Metabolism 200893 1526-1540. (doi:10.1210/ jc.2008-0125)

4 Petersenn S, Newell-Price J, Findling JW, Gu F, Maldonado M, Sen K, Salgado LR, Colao A \& Biller BMK. High variability in baseline urinary free cortisol values in patients with Cushing's disease. Clinical Endocrinology 201480 261-269. (doi:10.1111/cen.12259)

5 Friedman TC, Ghods DE, Shahinian HK, Zachery L, Shayesteh N, Seasholtz S, Zuckerbraun E, Lee ML \& McCutcheon IE. High prevalence of normal tests assessing hypercortisolism in subjects with mild and episodic Cushing's syndrome suggests that the paradigm for diagnosis and exclusion of Cushing's syndrome requires multiple testing. Hormone and Metabolic Research 201042 874-881. (doi:10.105 5/s-0030-1263128)

6 Wester VL \& van Rossum EFC. Clinical applications of cortisol measurements in hair. European Journal of Endocrinology 2015173 M1-M10. (doi:10.1530/EJE-15-0313)

7 Pereg D, Chan J, Russell E, Berlin T, Mosseri M, Seabrook JA, Koren G \& Van Uum S. Cortisol and testosterone in hair as biological markers of systolic heart failure. Psychoneuroendocrinology 201338 2875-2882. (doi:10.1016/j.psyneuen.2013.07.015)

8 Pereg D, Gow R, Mosseri M, Lishner M, Rieder M, Van Uum S \& Koren G. Hair cortisol and the risk for acute myocardial infarction in adult men. Stress 201114 73-81. (doi:10.3109/10253890.2010.511352)

9 Manenschijn L, Schaap L, van Schoor NM, van der Pas S, Peeters GM, Lips P, Koper JW \& van Rossum EF. High long-term cortisol levels, measured in scalp hair, are associated with a history of cardiovascular disease. Journal of Clinical Endocrinology and Metabolism 201398 2078-2083. (doi:10.1210/jc.2012-3663)

10 Chan J, Sauve B, Tokmakejian S, Koren G \& Van Uum S. Measurement of cortisol and testosterone in hair of obese and non-obese human subjects. Experimental and Clinical Endocrinology and Diabetes 2014122 356-362. (doi:10.1055/s-0034-1374609)

11 Wester VL, Staufenbiel SM, Veldhorst MA, Visser JA, Manenschijn L, Koper JW, Klessens-Godfroy FJ, van den Akker EL \& van Rossum EF. Long-term cortisol levels measured in scalp hair of obese patients. Obesity 201422 1956-1958. (doi:10.1002/oby.20795)

12 Veldhorst MA, Noppe G, Jongejan MH, Kok CB, Mekic S, Koper JW, van Rossum EF \& van den Akker EL. Increased scalp hair cortisol concentrations in obese children. Journal of Clinical Endocrinology and Metabolism 201499 285-290. (doi:10.1210/jc.2013-2924)

13 Stalder T, Kirschbaum C, Alexander N, Bornstein SR, Gao W, Miller R, Stark S, Bosch JA \& Fischer JE. Cortisol in hair and the metabolic syndrome. Journal of Clinical Endocrinology and Metabolism 201398 2573-2580. (doi:10.1210/jc.2013-1056)

14 Thomson S, Koren G, Fraser LA, Rieder M, Friedman TC \& Van Uum SH. Hair analysis provides a historical record of cortisol levels in Cushing's syndrome. Experimental and Clinical Endocrinology and Diabetes 2010118 133-138. (doi:10.1055/s-0029-1220771)

15 Manenschijn L, Koper JW, van den Akker EL, de Heide LJ, Geerdink EA, de Jong FH, Feelders RA \& van Rossum EF. A novel tool in the diagnosis and follow-up of (cyclic) Cushing's syndrome: measurement of long-term cortisol in scalp hair. Journal of Clinical Endocrinology and Metabolism 201297 E1836-E1843. (doi:10.1210/jc.2012-1852) 
16 Manenschijn L, Koper JW, Lamberts SW \& van Rossum EF. Evaluation of a method to measure long term cortisol levels. Steroids 201176 1032-1036. (doi:10.1016/j.steroids.2011.04.005)

17 Harkey MR. Anatomy and physiology of hair. Forensic Science International 199363 9-18. (doi:10.1016/0379-0738(93)90255-9)

18 Noppe G, Van Rossum EF, Koper JW, Manenschijn L, Bruining GJ, de Rijke YB \& van den Akker EL. Validation and reference ranges of hair cortisol measurement in healthy children. Hormone Research in Paediatrics 201482 97-102. (doi:10.1159/000362519)

19 Alwani RA, Schmit Jongbloed LW, de Jong FH, van der Lely AJ, de Herder WW \& Feelders RA. Differentiating between Cushing's disease and pseudo-Cushing's syndrome: comparison of four tests. European Journal of Endocrinology 2014170 477-486. (doi:10.1530/EJE-130702)

20 Elamin MB, Murad MH, Mullan R, Erickson D, Harris K, Nadeem S, Ennis R, Erwin PJ \& Montori VM. Accuracy of diagnostic tests for Cushing's syndrome: a systematic review and metaanalyses. Journal of Clinical Endocrinology and Metabolism 200893 1553-1562. (doi:10.1210/jc.2008-0139)

21 Zhang Q, Dou J, Gu W, Yang G \& Lu J. Reassessing the reliability of the salivary cortisol assay for the diagnosis of Cushing syndrome. Journal of International Medical Research 201341 1387-1394. (doi:10.1177/0300060513498017)
22 Short SJ, Stalder T, Marceau K, Entringer S, Moog NK, Shirtcliff EA, Wadhwa PD \& Buss C. Correspondence between hair cortisol concentrations and 30-day integrated daily salivary and weekly urinary cortisol measures. Psychoneuroendocrinology 2016 71 12-18. (doi:10.1016/j.psyneuen.2016.05.007)

23 Dettenborn L, Muhtz C, Skoluda N, Stalder T, Steudte S, Hinkelmann $\mathrm{K}$, Kirschbaum $\mathrm{C} \&$ Otte $\mathrm{C}$. Introducing a novel method to assess cumulative steroid concentrations: increased hair cortisol concentrations over 6 months in medicated patients with depression. Stress 201215 348-353. (doi:10.3109/10253890.2011.619239)

24 Russell E, Kirschbaum C, Laudenslager ML, Stalder T, De Rijke Y, van Rossum EF, Van Uum S \& Koren G. Toward standardization of hair cortisol measurement: results of the first international interlaboratory round robin. Therapeutic Drug Monitoring 201537 71-75. (doi:10.1097/FTD.0000000000000148)

25 Raff H \& Singh RJ. Measurement of late-night salivary cortisol and cortisone by LC-MS/MS to assess preanalytical sample contamination with topical hydrocortisone. Clinical Chemistry 201258 947-948. (doi:10.1373/clinchem.2012.182717)

26 Abell JG, Stalder T, Ferrie JE, Shipley MJ, Kirschbaum C, Kivimäki M \& Kumari M. Assessing cortisol from hair samples in a large observational cohort: the Whitehall II study. Psychoneuroendocrinology 201673 148-156. (doi:10.1016/j.psyneuen.2016.07.214)

Received 21 October 2016

Revised version received 18 February 2017

Accepted 13 March 2017 